\title{
Linear and non-linear amplification of high-mode perturbations at the ablation front in HiPER targets
}

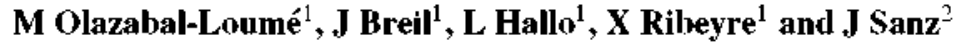 \\ ${ }^{1}$ CELIA, UMR 5107 Université Botdeaux 1-CNRS-CEA, 351 cours de la Libétation. \\ 33405 Talence, France \\ ${ }^{2}$ ETSI Aeronauticos. Universidad Politecnica de Madrid, Madrid 28040, Spain \\ E-mail: olazabal@ $@$ lia.u-bordeaux 1.fr
}

\begin{abstract}
The linear and non-linear sensitivity of the $180 \mathrm{~kJ}$ baseline HiPER target to highmode perturbations, i.e. surface roughness, is addressed using two-dimensional simulations and a complementary analysis by linear and non-linear ablative Rayleigh-Taylor models. Simulations provide an assessment of an early nonlinear stage leading to a significant deformation of the ablation surface for modes of maximum linear growth factor. A design using a picket prepulse evidences an improvement in the target stability inducing a delay of the nonlinear behavior. Perturbation evolution and shape, evidenced by simulations of the non-linear stage, are analyzed with existing self-consistent non-linear theory.
\end{abstract}

\section{Introduction}

Petawatt class lasers enable ignition energy to be delivered directly to the Inertial Confinement Fusion (ICF) target. This fast-ignition (FI) approach $[1,2]$ requires much less laser energy than conventional ignition schemes $[3,4]$ and enables high gain to be achieved. Early FI target designs used a low implosion velocity to mitigate hydrodynamic instabilities, such as the ablative Rayleigh-Taylor (ART) instability which occurs during the acceleration phase. In the FI scheme, a relatively low energy nanosecond laser enables compression and an intense picosecond laser delivers the energy to achieve ignition. This is the main feature of the European inertial fusion energy project HiPER [5]. One-dimensional designs of the HiPER baseline target and preliminary estimates of the linear ART instability growth are reported in [6-8]. A safety factor discussion for low mode perturbations $(l \leqslant 50)$, related to laser irradiation non-uniformities, is addressed in [9]. It is shown that a self-consistent numerical method provides accurate estimates for the perturbation growth at the ablation front and at 
deuterium-tritium (DT) interfaces. Analytical estimates can also be obtained by coupling the perturbed shock waves with the ablation front in a high aspect ratio target. The perturbation growth can thus be understood by combining direct numerical simulations with a model posttreatment for low mode numbers corresponding to large wavelength perturbations. These perturbations can be initiated, for example, by laser irradiation non-uniformities. A tolerance guideline on the laser irradiation was suggested and dominant mode numbers were identified.

Pursuing hydrodynamic stability studies for the HiPER baseline target, we address here the problem of high-mode perturbations $(l \geqslant 20)$ induced by shell roughness. This work is organized into four parts. Firstly, the one-dimensional (1D) base flow is described for both standard and adiabat shaping scenarios. The development of perturbations both in time and in space is then discussed with the support of $2 \mathrm{D}$ simulations. The ART instability growth is calculated and compared with previous results obtained from theory as well as simulations at the linear stage $[6,7]$. Direct simulations are carried out up to mode number 500 , and the resulting amplifications are cross-checked with embedded linear modeling. Here, contrary to the low wave numbers case, non-linear behavior has to be accounted for during shell acceleration. Finally, it is shown that, for high modes, the ART instability behaves separately from the spatial perturbation seeded during the shock transit time. It depends only on the amplitude achieved at the beginning of the acceleration. Numerical simulations with the $2 \mathrm{D}$ code CHIC [10-13] provide an accurate estimate of the linear perturbation growth and an assessment of the non linear stage. It is also shown that stabilizing schemes based on adiabat shaping [14] reduce the perturbation growth and delay the beginning of the non-linear phase. This makes it possible to address the problem of determining the admissible initial roughness spectrum for the baseline HiPER target. In the third part, numerical results are analyzed in depth with existing theoretical predictions. Good agreement is found between simulations and self-consistent theories up to the end of the weakly non-linear phase.

\section{One-dimensional flow of the $180 \mathrm{~kJ}$ baseline target}

\subsection{Standard pulse}

The baseline HiPER target is described in [6-8]. This all-DT target has a $1044 \mu \mathrm{m}$ external radius and a shell thickness of $211 \mu \mathrm{m}$. The main performance levels of the fuel assembly, without radiative processes, are summarized in table 1. Two standard laser pulse shapes have been designed. The first (figure $1(a)$ ) takes account of the laser absorption via ray-tracing techniques, with $180 \mathrm{~kJ}$ laser energy. The second laser pulse (figure $\mathrm{I}(b)$ ) has been reshaped from the first to obtain the same overall performance levels presented in table 1 , but assuming total absorption at the critical density and normal laser ray incidence. In this case, the laser energy is approximately $105 \mathrm{~kJ}$ with a maximum power of $26 \mathrm{TW}$. This design was studied in terms of low mode stability in [9] and was recently used in shock ignition studies $[15,16]$. A set of studies for this target is presented in [17], including shock ignition and fast ignition applications. Total laser absorption and a normal incidence approach was used in [18] to perform 2D single-mode calculations for modes $l \geqslant 20$. Figure 2 shows the $r-t$ diagram of the temporal evolution of the target shell during laser pulse interaction. The diagram presents the target radius in time. The shock, due to the laser pulse foot, breaks out of the shell around $7 \mathrm{~ns}$. The shock level is set to compress the target on a low adiabat, i.e. close to 1 (see table 1). The shell is compressed, accelerated and the fuel assembly is completed (around $11 \mathrm{~ns}$ ) at the stagnation phase, where the density and the areal density reach their maxima. 
Table 1. Target performance levels of the HiPER baseline target. $\left(P_{\text {Fermi }} \simeq 2.2 \rho^{5 / 3}\right.$ (Mbar) and $P, \rho$ : shell pressure and density, tespectively).

\begin{tabular}{ll}
\hline Initial aspect ratio & $\simeq 5$ \\
IFAR (in-flight aspect ratio) & 35 \\
In-flight mass $(\mathrm{mg})$ & $\simeq 0.28$ \\
Implosion velocity $\left(\mathrm{cm} \mathrm{s}^{-1}\right)$ & $\simeq 2.9 \times 10^{7}$ \\
Adiabat $\alpha=P / P_{\text {Ferni }}$ & $\simeq 1.0$ \\
Peak areal density $\rho R\left(\mathrm{~g} \mathrm{~cm}^{-2}\right)$ & $\simeq 1.50$ \\
Peak density $\left(\mathrm{g} \mathrm{cm}^{-3}\right)$ at peak $\rho R$ & $\simeq 740$ \\
\hline
\end{tabular}
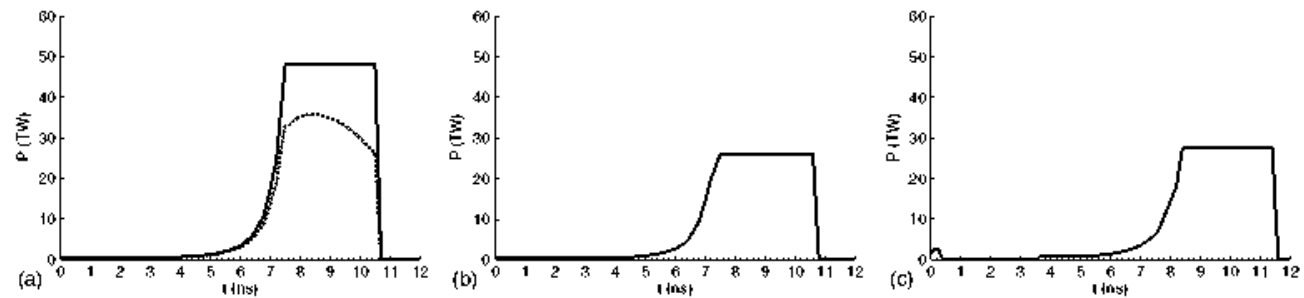

Figure 1. (a) Laset pulse shape with ray-tracing: incident laser energy $E_{\mathrm{L}}=180 \mathrm{~kJ}$ (solid line), absorbed laser energy $E_{\mathrm{a}} \simeq 126 \mathrm{~kJ}$ (dotted line) and absorbed energy rate $\eta_{0} \simeq 70 \%$. (b) Laser pulse shape with total absorption at critical density and normal incidence: $E_{\mathrm{L}}=E_{\mathrm{a}} \simeq 105 \mathrm{~kJ}$, $\eta_{\mathrm{A}}=100 \%$ (c) 'Adjabat shaping' laser pulse shape with total absorption at critical density and normal incidence: $E_{\mathrm{L}}=E_{\mathrm{A}} \simeq 105 \mathrm{~kJ}, \eta_{\mathrm{a}}=100 \%$.

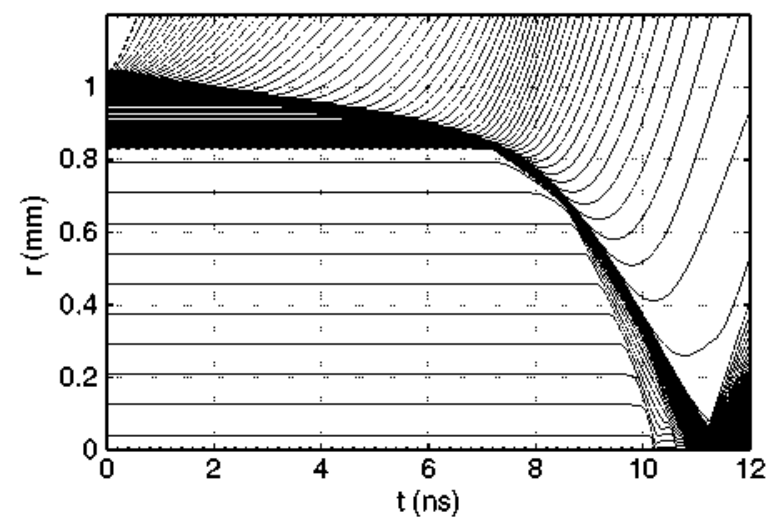

Figure 2. $r-t$ diagram of the HiPER baseline target with the standard pulse shapes $1(a)$ and $(b)$.

\subsection{Adiabat shaping design using totally absorbed energy}

The laser pulse shape presented in figure $1(c)$ consists of a low intensity picket and a period when the laser is turned off and finally the main pulse, providing the adiabat shaping by relaxation [14]. Once again, total absorption of laser energy at the critical density is assumed. For this design, acceleration, implosion velocity and inner adiabat, maximum density and maximum areal density are similar to the standard case, while a higher ablation velocity $(+30 \%)$ makes it possible to reduce the ART instability growth during the main acceleration. The shock breaks out and the main acceleration occurs with a $900 \mathrm{ps}$ delay. It makes it possible to start the main pulse (pulse foot) at a higher laser power level and at a reduced contrast ratio 

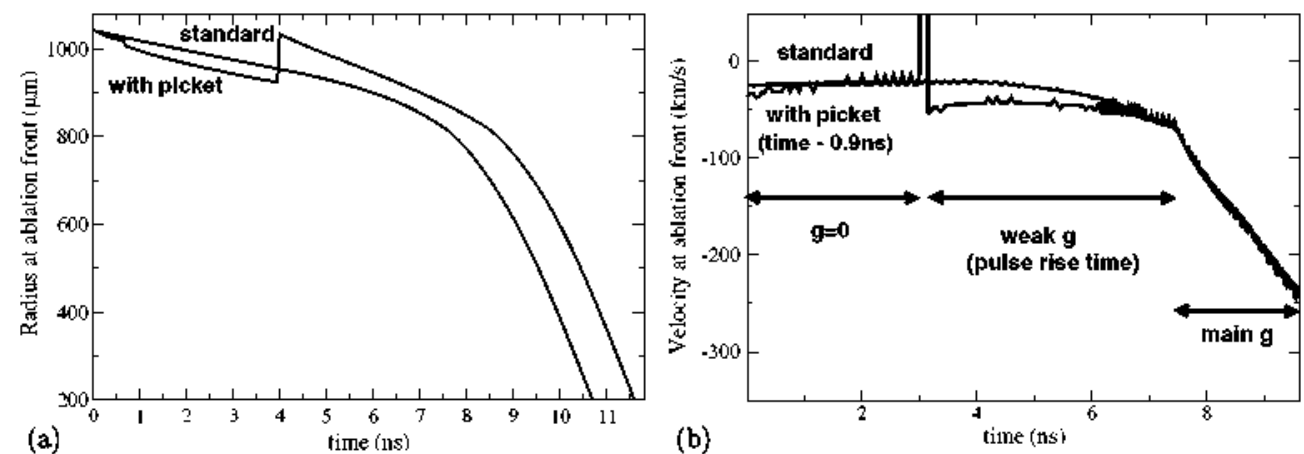

Figure 3. Ablation front radius $(a)$ and velocity $(b)$ versus time. Three distinct regimes are identified versus the acceletation $g$ : (1) the formation-propagation of the ablation front $(g=0$ ), (2) the weakly accelerated regine due to laser power increase (weak $g$ ) and (3) the main accelerated tegime (main $g$ ).

of the maximum power to the foot power. The next section shows that this pulse shape also improves the target stability.

\subsection{Comparison of the 10 flow and acceleration phase definition}

Figure 3 presents the 1D ablation front dynamics: the radius and the velocity versus time for the standard and adiabat shaping designs. Note that in the adiabat shaping case, the discontinuity in the radius curve around $4 \mathrm{~ns}$ corresponds to the ablation zone created by the launch of the second shock, at the beginning of the main pulse. The velocity curve for the picket case is shifted by $900 \mathrm{ps}$ for an easier comparison with the standard case. With the standard design dynamics, three zones can be distinguished: a constant velocity $(t<3.5 \mathrm{~ns})$, a weak acceleration (3.5-7.5 ns) corresponding to the laser power rise, and the main acceleration (7.5-9.5 11s) after the shock break out. In the picket case, the first and third zones are similar to the standard case. The reduced contrast ratio enables us to keep an approximately constant velocity in the second zone, i.e. to reduce the total acceleration time.

\subsection{Estimates of the growth factor}

The growth factor is obtained from the integration in time of the theoretical growth rate formula $\gamma=\alpha \sqrt{k g}-\beta k V_{a}$ given by [19] using the coefficients for DT: $\alpha=0.94, \beta=2.7$, where $g$ is the acceleration at the ablation front, $V_{a}$ the ablation velocity and $k=1 / r$ the wave number with $r$ the radius at the ablation front and $l$ the mode number. The integration domain includes both 'weak' and 'main' acceleration zones. Figure 4 presents the mean growth factor versus the mode number with and without the picket. It shows that it is reduced by a factor of 1.8 when a picket is used. The mode number where maximum amplification is observed is not greatly modified by the picket, but the maximum growth factor is decreased from 9 to 5 which suggests that the non-linear regime may be suppressed or at least delayed. Results of $2 \mathrm{D}$ simulations plotted for this period are in good agreement with theoretical predictions. Details of the simulations are given in section 3 . These results are consistent with $[6,7]$. In [6], the $135 \mathrm{~kJ}$ design was studied and theoretical growth factors were obtained. In [7], growth factors were calculated with a linear stability code [20] and compared with analytical models [19], considering the main acceleration period. The results obtained for the $180 \mathrm{~kJ}$ design give equivalent growth factors with a maximum of 9 for the standard pulse (approximately 7 is 


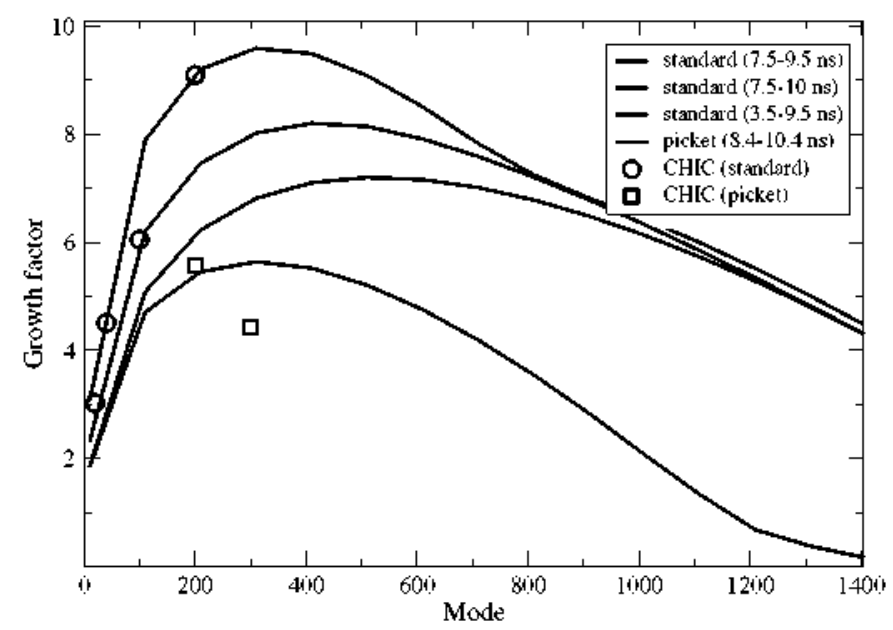

Figure 4. Growth factor versus mode number, for standard and picket pulse shapes (linear theory and C.HIC calculations). The maximum is reduced by a factor of 1.8 using the adiabat shaping. For the standard case. the contribution of different periods is shown.

obtained integrating only on the main acceleration time), and it is lower than 5 for the adiabat shaping design. The curves corresponding to the integration on the range $7.5-9.5 \mathrm{~ns}$ and 7.5-10 ns are also plotted in figure 4 to show the contribution of the different periods to the perturbation growth. Considering high values of this factor obtained for the standard case, we performed a set of full 2D simulations of the perturbed flow to estimate the non-linear effects for modes of high growth factor $l=200-300$.

\section{2D single-mode simulations of perturbed flow}

\subsection{Characteristics of the hydrodynamic ALE CHIC code}

CHIC is a 2D ALE (arbitrary Lagrangian Eulerian) integrated ICF code developed at CELIA. The numerical method for Lagrangian hydrodynamics is based on a recent numerical scheme [10]. This scheme uses a cell-centered discretization based on a total energy formulation. The velocity of the vertices and the fluxes are evaluated using a nodal solver, accounting for conservation laws and one entropy inequality. It can be viewed as a two-dimensional extension of the acoustic Godunov's solver which uses Riemann invariants at all faces around the node. It satisfies naturally the geometric conservation law. The high order extension [1 1, 12] uses piecewise linear reconstruction. ALE computations are performed using a cell-centered remapping procedure.

The code includes the ion and electron heat conduction, the thermal coupling of electrons and ions and a multigroup diffusion model for the radiation transport. The ionization and the opacity data are tabulated, assuming a local thermodynamic equilibrium (LTE) or a non-LTE depending on the plasma parameters. The radiative transport is computed assuming that the radiation field is quasi-stationary and weakly anisotropic. The QEOS [23] and SESAME [24] equations of state are available in the code. The laser propagation is computed via a ray-tracing algorithm which includes the refraction and collisional absorption.

To study the perturbation evolution at the ablation front, a Legendre single-mode perturbation is imposed at the external surface. This perturbation is radial and, because of 
the relatively small range of the spatial perturbation dependence, a linear decay inside the shell is used. This approximation preserves the ART seeding and growth. A purely Lagrangian computation may stop before the end of acceleration, during the non-linear stage due to tangled mesh. The ALE method [13] enables us to improve the geometric quality of the grid elements and to optimize accuracy, robustness and computational efficiency. A special rezoning procedure is implemented in the ablation zone. A traditional mesh smoothing is not sufficient in our case as it may decrease the accuracy of the Lagrangian computation with a consequent loss of spatial resolution. The Lagrangian displacement must be preserved at the ablation front. In the rezoning procedure implemented in CHIC, the Lagrangian characteristic of the mainstream flow and especially the perturbation at the ablation front are preserved using an orthoradial projection. In this rezoning procedure, the radial Lagrangian displacement of the nodes is kept during all the computation and the orthoradial displacement is projected orthogonally onto this radius. The rezoning phase is followed by a second order remapping phase where all the conservative quantities are conservatively remapped from the Lagrangian mesh onto this rezoned mesh. Once the non-linear perturbation growth has started, an additional mesh smoothing is added to this rezoning to avoid mesh tangling. By using such rezoning, a nonlinear evolution of mode numbers up to $l=800$ can be studied by using 35 grid points per wavelength.

\subsection{Low mode perturbations from irradiation non-uniformities and surface roughnes's}

Irradiation non-uniformities as well as surface roughness may produce and feed perturbations in the target. The most unstable regime occurs in the acceleration period (figure 3 ) where the perturbation can be seeded in the target due to spatial dependences. The initial perturbed state at the beginning of the main acceleration involves a very complex process. The low wave number perturbations can be fed through external surface roughness of the target and radiation non-uniformities. The ablation front may also transport initial perturbations for all wave numbers. Because of these different initialization mechanisms, mainly due to a spatial extension of perturbations, the spatial shape of the perturbation at the beginning of the main acceleration phase may be very different, especially for low wave numbers with wavelengths close to the target thickness where a strong spatial coupling is expected.

The ablation front perturbation history versus time is presented in figure 5 , for the mode $l=20$, up to the fuel assembly time. The spikes in the curves are due to the post-treatment of numerical data and may occur when the data are given at a time corresponding to a change of mass element (Lagrangian mesh) of the ablation front. The perturbation is initiated by a surface roughness or by irradiation non-uniformities (see [9]). The ART instability growth is similar even if the perturbation history before the main acceleration is different. That means that the ablation front perturbation is not affected by the perturbation spatial shape, by compressible effects and by the perturbation coupling to the shock wave. This is true at least for the mode number $l=20$ or higher. Moreover, from previous studies [9], it can be observed that this is not always true for $l<16$ where such a perturbation post-treatment should be performed with care because of strong spatial coupling.

\subsection{High-mode simulations}

3.3.1. Standard pulse. Figure 6 presents a cross-check validation of high-mode instantaneous linear growth rates obtained in CHIC simulations and from theory [19]. This comparison is made for short times up to $8 \mathrm{~ns}$, because of the early non-linear stage, which occurs for modes higher than $l=100$ including small initial perturbation amplitudes. Indeed for $l=100$ and a 


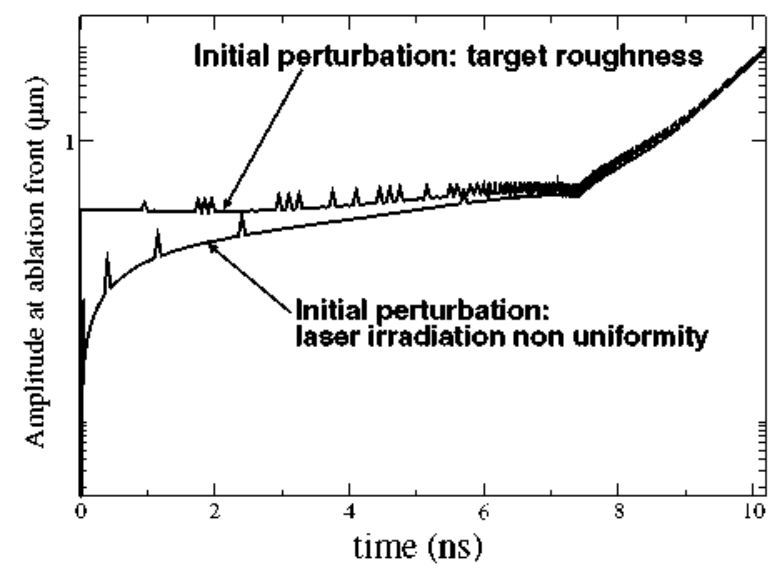

Figure 5. Perturbation amplitude versus time at the ablation front for a $t=20$ mode due to irradiation non-uniformities and surface roughness. The most unstable phase begins at time $t \approx 7.5 \mathrm{~ns}$, i.e. the beginning of the main acceletation. Both petturbations have the same ART instability growth, independent of their origins or of their spatial shapes.

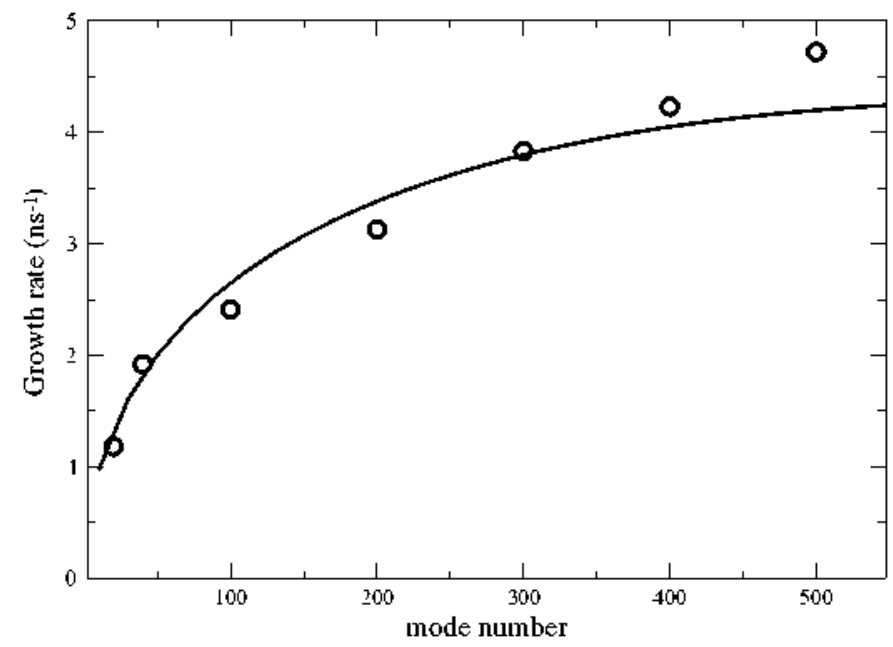

Figure 6. Linear growth rate at short times (7.5-8 ns) versus mode number obtained from the CHIC simulations (dots) compared with theoretical model (solid line) [19].

$0.1 \mathrm{~cm}$ radius, the non-linear threshold $a k \sim 0.1$ [25] is expected to be reached, for amplitudes larger than $1 \mu \mathrm{m}$, within the nanosecond time scale. It should be noted that at the beginning of acceleration, most unstable modes are in the range [500-600] as the maximum growth factor is obtained for modes in the range [200-300]. It shows a substantial time dependence in the perturbation development for the linear and non-linear regimes, including the contribution of at least the second harmonic.

Figure 7(a) shows the perturbation amplitude at the ablation front versus time for different initial amplitudes with an initial single-mode perturbation $l=200$. The transition to the non-linear stage is indicated by the change in the slope during evolution of the amplitude. The perturbation evolves in the linear regime (see figure $7(b)$ ) and then reaches the weakly 
(a)

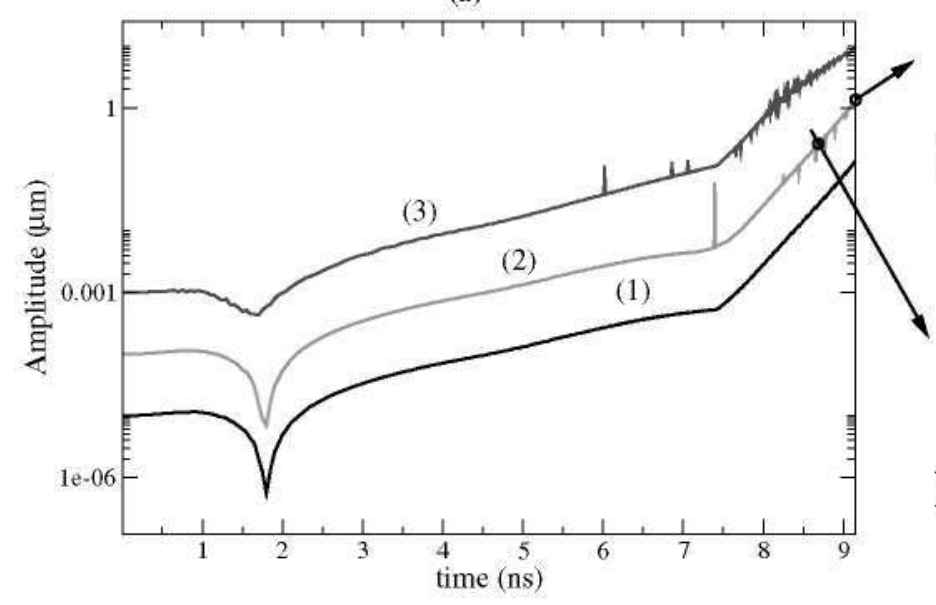

(c)

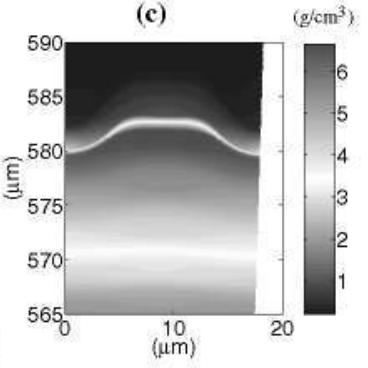

(b)

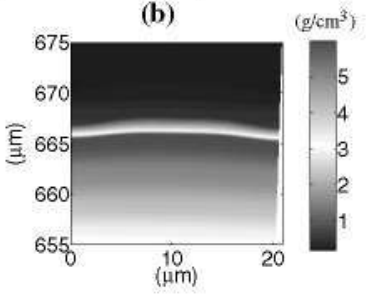

Figure 7. (a) Ablation front perturbation versus time for $l=200$ mode with three different initial amplitudes and density contour shapes of curve (2) at times (b) $8.7 \mathrm{~ns}$ and $(c) 9.2 \mathrm{~ns}$. On density contours, the blue zone at the top is the external vacuum, the red zone is the compressed DT and the blue zone at the bottom is the vapor-pressure DT gas in the center.

non-linear regime (figure $7(c)$ ). At that moment, the particular shape of density contours is characteristic of the ART instability behavior. Here at least a double frequency structure is present. The effect of ablation on the perturbation shape during the weakly non-linear regime is clearly seen. This shape is described and explained in $[21,22]$. The spikes may flatten and enlarge due to the dynamic ablation overpressure. In this regime, the perturbation shape is the result of early seeding of harmonics and their feedback to the fundamental mode. These harmonics can appear with the same or opposite phase relative to the fundamental mode [21]. Figure $8(a)$ shows the amplitudes (absolute values) of the first and second harmonics as a function of time. Figure $8(b)$ shows a Fourier analysis of the modal spectrum during the main acceleration, at time $t=9.2 \mathrm{~ns}$, up to the fifth harmonic. The shape of the density contours (wide and flattened) in figure $7(c)$ is due to the phase inversion of the second harmonic (figure $8(b)$ ).

The different shapes of the density contours for $l=200$ are shown in figure 9 at different times excited by the initial roughness of about $1 \mathrm{~nm}$ (figures $(a)-(c)$ ). A peak-and-valley shape can be seen up to the fully non-linear regime at the time $t>9 \mathrm{~ns}$. The non-linear shape consists of spikes and bubbles. At the end of the simulation, multi-frequency structures appear. For an initial amplitude of a few nanometers, the non-linear evolution lasts during most of the acceleration phase and non-linear couplings may be very strong. For a higher initial amplitude of around $10 \mathrm{~nm}$ or more, characteristic structures appear with very singular shapes (see figure 9(e)), previously observed in planar simulations in [21]. At a later time, an asymptotic case is achieved, where the shell is completely decompressed (figure $9(f)$ ). These initial conditions produce very complex multimode structures of the non-linear regime, where ablation plays an important role. The shape of the perturbation differs from the classic case where thin spikes and thick bubbles are expected [21]. This new distribution is clearly observed for higher modes in our simulations. In figure 10 , for mode $l=300$, the clear inversion of the shape perturbation (thin bubbles, thick spikes) from the weakly non-linear stage can be seen (figure $10(a)$ ). This behavior has been observed for all higher modes. Theory 

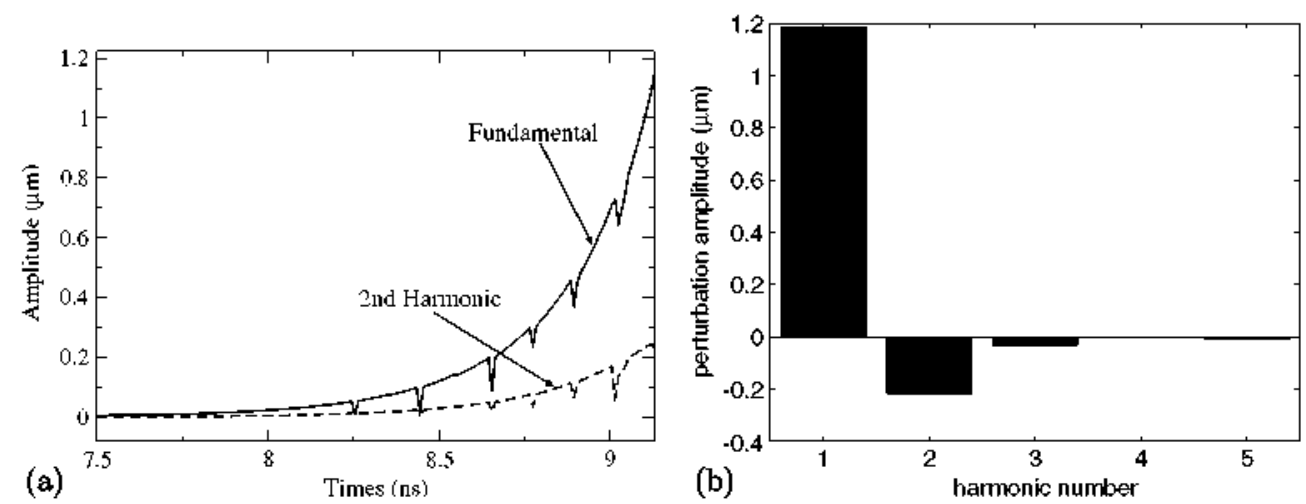

Figure 8. For mode $l=200:(a)$ anplitude of the first and second harnonics versus time during the acceleration phase; (b) Foutier analysis of the ablation ftont petturbation at time 9.2 ns acceleration. up to the fifth harmonic. The second harmonic appears with the opposite phase.

gives a threshold wave number for this feedback. Our simulations, initiated by a single-mode perturbation $(l=300,500,800)$, reproduce the inversion of spikes and bubbles thickness, contrary to a classic Rayleigh-Taylor (RT) weakly non-linear perturbation shape, i.e. without ablation. Simulation results are consistent with the existence of this threshold and all unsteady effects. For example, the dominant mode in the linear regime differs at the beginning of the main acceleration and subsequent times. For this reason, the non-steady effects act on the growth of both fundamental and harmonics of the initial perturbation. The initial amplitude may also change the contribution of these harmonics. At later times, the perturbation evolves in the non-linear regime with a multimode bubble-spike structure (figure 10(b)). Non-linear effects at the ablation front produce very elongated bubbles which extend from the ablation front into the expanding low density plasma (figure 10(c)). This behavior will be discussed in section 4.

Figure 11 shows the temporal evolution of the perturbation amplitude at the ablation front for the mode $l=300$ and for three initial amplitudes. The behavior is the same before the main acceleration phase as long as the perturbation remains in the linear stage. The slope variation indicating the non-linear perturbation behavior occurs for the same amplitudes. The $Y$ curves indicate fraction levels of the perturbation wavelength $\lambda$. In particular, $Y=0.1 \lambda$ corresponds to the classical weakly non-linear saturation criteria [25]. For the mode $l=300$, we checked that the radiation transport has little influence on the instability growth. At the end of acceleration, the radiation changes the amplitude by less than $10 \%$. For modes greater than 300 , shape inversion was observed, with thicker spikes. In addition, for these modes, the perturbation may reverse phase during the weakly non-linear stage.

33.2. Adiabat shaping pulse. 2D simulations were also performed using the adiabat shaping laser pulse. Figure 12 shows the perturbation amplitude at the ablation front for $l=300$. The perturbation oscillates with different frequencies before and during the laser pulse rise time (shock transit regime). ART instability develops at a later time. The final amplitude is considerably smaller (with approximately a factor of 300 ) than the one found in the standard case with the same initial amplitude (figure 11, curve 2). This result confirms the reduction in the growth factor shown by theoretical estimates in section 1, and takes account of the different behavior before the main acceleration. 